\title{
Evodiamine Induces Extrinsic Apoptosis Through P38 Mapk Pathway and Inhibits Autophagy in Gallbladder Cancer
}

\author{
Jun jie $\mathrm{Xu}^{1}$, Ran Yang ${ }^{1}$, Fang jing Yang ${ }^{1}$ and Ying bin Liu ${ }^{2,3 *}$ \\ ${ }^{1}$ Shanghai Jiao Tong University School of Medicine, China \\ ${ }^{2}$ Department of General Surgery, Xinhua Hospital affiliated to Shanghai Jiao Tong University School of Medicine, China \\ ${ }^{3}$ Institute of Biliary Disease Research, Shanghai Jiao Tong University School of Medicine, China
}

Received: 阱 June 22, 2018; Published: 阱July 05, 2018

*Corresponding author: Ying-bin Liu, Department of General Surgery, Xinhua Hospital affiliated to Shanghai Jiao Tong University School of Medicine\& Institute of Biliary Disease Research, Shanghai Jiao Tong University School of Medicine, N0.1665 Kongjiang Road, Shanghai, China

\begin{abstract}
The gallbladder cancer is the most common malignant tumor of the biliary tract and the third most common gastrointestinal malignant tumor in the world for public health with extremely poor prognosis. The primary purpose of this study is to investigate the inhibition effects of evodiamine, a major alkaloid compound extracted from the dry unripened fruit Evodiae fructus, on the carcinoma of the gallbladder cancer, and explored the underlying molecular mechanisms responsible for these effects. Cell viability of NOZ and GBC-SD cells was assessed by CCK-8 and colony forming assays. Cell apoptosis was analyzed by Annexin V-FITC and PI double staining flow cytometry and the apoptosis-related DNA damage was detected by Immunofluorescence microscopy assays. Western blot analysis was used to analyze the expression of crucial proteins involved in apoptosis, autophagy and signaling pathways. In this study, we found evodiamine induced cell viability and colony forming inhibition, apoptosis promotion and autophagy inhibition of NOZ and GBC-SD cells in a dose-dependent manner. The activation of p38 MAPK signaling pathway was significantly associated with evodiamine-treated apoptosis of gallbladder cancer cells, and the depletion of p53 was related with decreasing autophagy. Consequently, we propose that evodiamine may serve as a potential therapeutic agent for gallbladder cancer.
\end{abstract}

Keywords: Evodiamine; Gallbladder Cancer; Apoptosis; P38 MAPK Pathway; Autophagy

Abbreviations: EVO: Evodiamine; C-caspase3 \& C-caspase8: Cleaved caspase-3, Cleaved caspase-8; P38 MAPK pathway: p38 Mitogen Activated Protein Kinase Pathway; LC3 II:Microtubule-Associated Protein 1 light 3 II

\section{Introduction}

Gallbladder cancer is the most common malignancy of the biliary tract. It is a lethal disease for most patients with a very poor prognosis and the 5 -year survival rate is less than $5 \%$ 10\% [1-3]. The etiology of gallbladder carcinoma remains poorly understood, yet the epidemiological studies have showed great disparities in geography, ethnic and gender [4,5]. Due to its inconspicuous signs and vague symptom, early diagnosis is hard and thus surgical resection is the only potentially curative therapy for many patients. Nevertheless, most of these patients suffer from recurrence or failure of the operation and require more radical therapy [6]. For these patients and those with unresectable gallbladder carcinoma, chemotherapy and radiotherapy are the remaining options, however, the therapeutic effect is not satisfactory at present. Accordingly, novel therapeutic strategies and potential anticancer drugs against gallbladder cancer are urgently needed. Evodiamine (EVO, Figure $1 \mathrm{~A}$ ) is a major alkaloid compound extracted from the dry unripened fruit Evodiae fructus, which has been found to be effective in the treatment of metabolic disorders, neurological disorders, and cardiovascular disorders $[7,8]$.

Recent researches have paid more attention to its anticancer activity. It's identified that EVO exerted inhibitory effects on certain kinds of cancers, including human ovarian cancer, hepatocellular carcinoma, leukemia by anti-proliferative and apoptosis-inducing activities [911]. Studies have shown that the EVO-induced inhibition effects on cancer is relevant to cell cycle arrest [12-15], apoptosis [9,16-20], autophagy $[21,22]$, and the regulation of certain signaling pathways, such as the suppression of JAK2-STAT3 signaling pathway $[23,24]$ and the down-regulation of PI3K-Akt pathway [25,26]. Apart from all these findings, the effect of EVO on gallbladder cancer has not been investigated yet, and the potential mechanisms involved remain unknown. In this study, we investigated the EVO-induced inhibition effects on gallbladder cancer and explored the underlying 
molecular mechanisms responsible for these effects, which could natural anti-tumor medicine for the gallbladder cancer. provide experimental evidence for the applying of EVO as a new

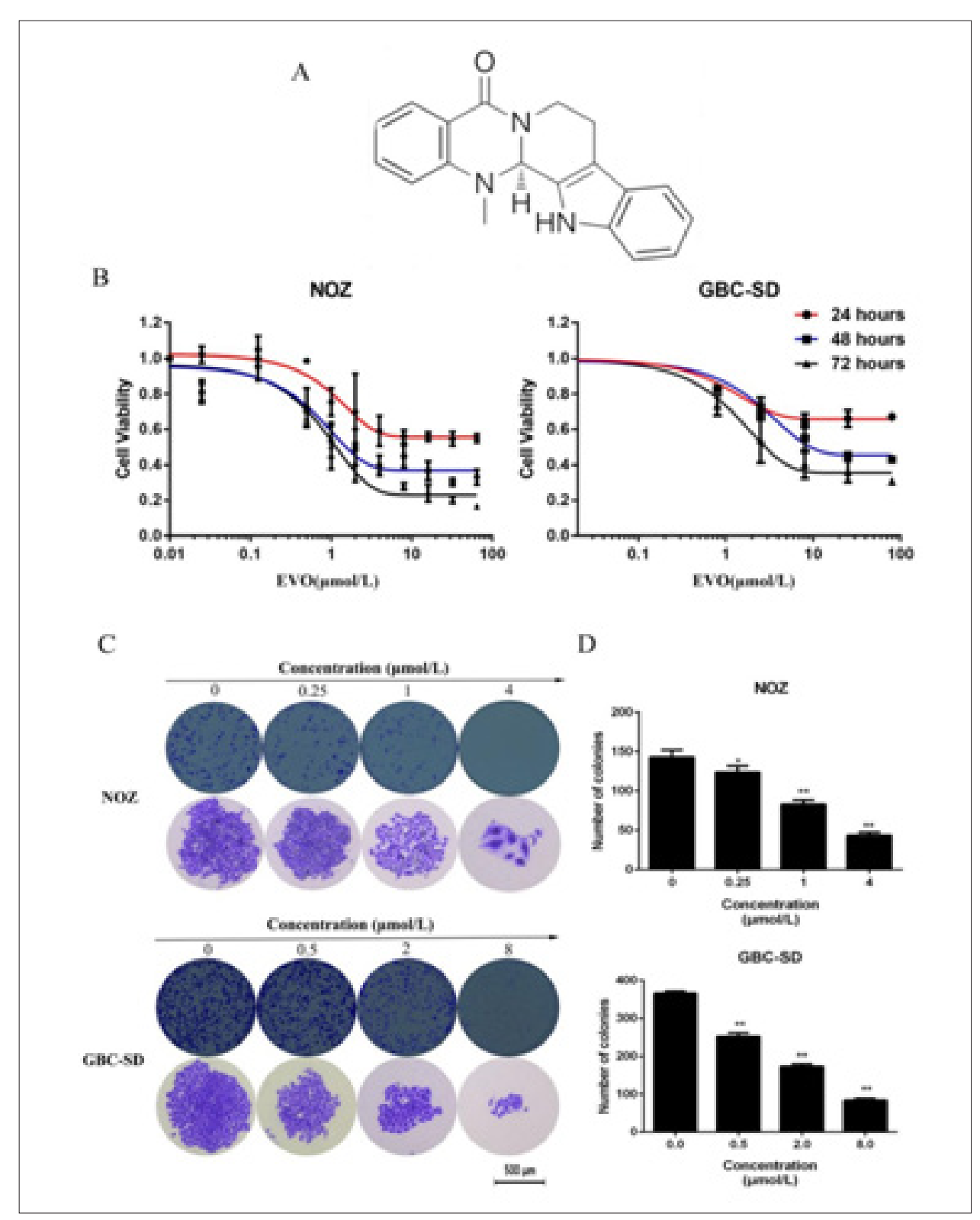

Figure 1: EVO inhibited the proliferation and colony formation of NOZ and GBC-SD cells.

\section{Materials and methods}

\section{Chemicals and reagents}

EVO was purchased from CHENGDU MUST BIO-TECHNOLOGY CO,LTD and dissolved in dimethyl sulfoxide (DMSO) to create a stock solution $(0.1 \mathrm{mmol} / \mathrm{L})$, which was stored at $-20^{\circ} \mathrm{C}$. The stock solution was further diluted with culture medium to yield the desired concentration. The DMSO concentration was kept below $0.1 \%$ in cell culture and no detectable effect on cell growth or cell death were observed. Cell Counting Kit-8 (CCK8), Histone H2A.X(phosphoS139) Elisa Kit were purchased from Abcam(ab2893). AnnexinV/ PI Apoptosis Kit was purchased from Invitrogen (Carlsbad, CA, USA).

\section{Cell lines and culture}

The cell lines NOZ and GBC-SD were purchased from the Cell Bank of Type Culture Collection of Chinese Academy of Sciences (Shanghai, China). NOZ cells were cultured in William's medium, and GBC cells were cultured in DMEM medium. The media for the cell lines were supplemented with 10\% fetal bovine serum (Gibco), $100 \mu \mathrm{g} / \mathrm{mL}$ streptomycin, and $100 \mathrm{U} / \mathrm{mL}$ penicillin (Hyclone, Logan, UT, USA) and maintained at $37^{\circ} \mathrm{C}$ in a humidified atmosphere with $5 \% \mathrm{CO} 2$.

\section{Cell viability assay}

A CCK-8 assay was used to evaluate NOZ and GBC-SD cell viability. Cells were seeded into 96-well plates at a density of 
2000 cells /well and were cultured for approximately 24h. Various concentrations of EVO were subsequently added, and the cells were incubated for $24 \mathrm{~h}, 48 \mathrm{~h}$ or $72 \mathrm{~h}$.Then the CCK-8 $(10 \mu \mathrm{l})$ was added and incubated for $2 \mathrm{~h}$ in the dark. Absorbance was measured at $450 \mathrm{~nm}$ using a microplate reader (Bio-Tek, Winooski, VT, USA). IC50 was measured by Graphpad Prism 5.

\section{Colony formation assay}

NOZ and GBC-SD cells were plated into a 6-well culture plate (500 cells/well) and allowed to adhere for $24 \mathrm{~h}$. After adherence, NOZ cells were treated with EVO $(0,0.025,0.1,0.4 \mu \mathrm{mol} / \mathrm{L})$ and the GBC cells with EVO $(0,0.05,0.2,0.8 \mu \mathrm{mol} / \mathrm{L})$. The cells were allowed to form colonies in complete medium for 7 days. Then, the colonies were fixed with $4 \%$ paraformaldehyde for $15 \mathrm{~min}$, stained with $0.1 \%$ crystal violet (Sigma-Aldrich) for $30 \mathrm{~min}$. After washing, the plates were air-dried, and the stained colonies were photographed using a microscope (Leica, Wetzlar, Germany). The total number of colonies ( $>50$ cells/colony) was counted manually.

\section{Cell apoptosis assay}

The GBC-SD and NOZ cells were treated with EVO for $48 \mathrm{~h}$. Adherent cells were then harvested by trypsinization, and floating cells were also harvested. After washing twice with cold PBS, the cells were resuspended at a density of $1 \times 106$ cells/mL. Next, 100 $\mu \mathrm{L}$ of binding buffer containing $5 \mu \mathrm{L}$ of Annexin V-FITC and $5 \mu \mathrm{L}$ of PI working solution $(100 \mu \mathrm{g} / \mathrm{mL})$ was added to the cells, followed by incubation for $15 \mathrm{~min}$ in the dark. Finally, the samples were then immediately analyzed by flow cytometry (BD Biosciences, San Diego, CA, USA).

\section{Immunofluorescence}

GBC-SD cells were treated with EVO for $48 \mathrm{~h}$. After washed with PBS, the cells were fixed with cold $4 \%$ paraformaldehyde for $30 \mathrm{~min}$ and then washed again with PBS. Triton X-100 (0.05\%) was added for $5 \mathrm{~min}$ to permeabilize the cells. The cells were blocked with bovine serum albumin then incubated with the primary antibody, after washed with PBS, the cells were incubated with rabbit antihuman $\gamma$-H2AX (Abcam, ab2893) conjugated with fluorescein isothiocyanate for 1 hour at room temperature. The cells were then washed again with PBS and stained with DAPI (4',6-diamidino2-phenylindole) and examined on a microscope (Leica, Wetzlar, Germany).

\section{Western blot analysis}

The GBC-SD cells and NOZ cells were treated with EVO for 48 h. Then, the cells were lysed in a RIPA buffer (Beyotime Institute of Biotechnology, Shanghai, China) and protease inhibitor (Roche Applied Science, Indianapolis, IN, USA). Protein con-centration was determined using the BCA Protein Assay (Beyotime Institute of Biotechnology). Protein samples were separated using sodium dodecyl sulfate polyacrylamide gel electrophoresis and transferred to polyvinylidene difluoride membranes (Millipore, Bedford, MA, USA). Membranes were then incubated with respective primary antibodies, followed by HRP-conjugated secondary antibodies. Proteins were visualized using the Gel Doc 2000 (Berkeley, California, USA). $\beta$-actin antibody was used as an internal control for whole cell lysates. The antibodies used which is related with apoptosis were Bax, Bcl-2, cleaved caspase-3, cleaved caspase-8,cleaved poly(ADPribose) polymerase (PARP), P38, p-P38(Thr180/Tyr182).

\section{RNA extraction and real-time quantitative RT-PCR}

NOZ and GBC-SD cells were plated into a 6-well culture plate (105 cells/well) and allowed to adhere for $24 \mathrm{~h}$ before treatment. After adherence, NOZ cells were treated with EVO for $24 \mathrm{~h}$. Then total RNA was extracted according to the manufacturer's instructions (TRIzol, Invitrogen, USA). RNA ( $2 \mu \mathrm{g}$ ) was reverse transcribed into cDNA (PrimeScript RT Master Mix (RR036A Takara)). Quantitative TNF- $\alpha$ mRNA levels were assessed using SYBR ${ }^{\circledR}$ Premix Ex Taq ${ }^{\text {TM }}$ (RR420A Takara) according to the manufacturer's protocol. GAPDH was used as an internal control. The qPCR primer sequences were as follows: TNF- $\alpha$, F5'-CCTCTCTCTAATCAGCCCTCTG3', R 5 ' - G A G G A C C T G G G A G TA GAT G A G - 3 '; G A P D H , F 5 ' ACTTTGGTATCGTGGAAGGAC, R5'-GGATGATGTTCTGGAGA-GCC. Conditions were as follows: $95^{\circ} \mathrm{C}$ for 30 s, then 40 cycles of $95^{\circ} \mathrm{C}$ for $5 \mathrm{~s}, 60^{\circ} \mathrm{C}$ for $30 \mathrm{~s}$. Each reaction was performed in triplicate.

\section{Statistical analysis}

All data and results were confirmed in at least 3 independent experiments. The results of each experiment are presented as mean \pm SD or as indicated. Student's t-test was used to compare the difference between treated groups and their controls using SPSS version 19.0 software (IBM Corporation). A P-value of less than 0.05 was considered significant for all tests.

\section{Results}

\section{EVO inhibited viability of NOZ and GBC-SD cells}

The CCK-8 was carried out to test cell viability. Treatment with EVO resulted in a marked decrease in viability of NOZ and GBC-SD cells in a dose-dependent and time-dependent manner (Figure 1B). The IC50 values (the concentration of drug inhibiting $50 \%$ of the cells) of NOZ and GBC-SD cells at $48 \mathrm{~h}$ were around 0.8 and 1.5 $\mu \mathrm{mol} / \mathrm{L}$. According to the viability curve, we chose $0,0.25,1,4 \mu \mathrm{mol} / \mathrm{L}$ and $0,0.5,2,8 \mu \mathrm{mol} / \mathrm{L}$ as the optimum concentration respectively for NOZ and GBC-SD cells in the following experiments. To investigate the independent growth of cells, the ability of GBC-SD and NOZ cells in the presence of EVO to form colonies was assessed by the flat plate colony formation assay (Figure 1C). The colony count indicated that EVO induced a dose-dependent decrease in colony formation. The numbers of colonies of EVO-treated GBC-SD and NOZ cells was significantly lower than those in the control group (Figure 1D). These findings demonstrated that EVO significantly inhibited the viability and proliferation of NOZ and GBC-SD cells.

\section{EVO induced apoptosis in GBC-SD and NOZ cells}

We used annexin V-FITC /PI staining and flow cytometry to examine whether EVO-induced growth inhibition was a result of apoptosis. As shown in (Figure 2A), EVO induced a dosedependent increase in the percentage of apoptotic cells and the dose of $4 \mu \mathrm{mol} / \mathrm{L}$ for NOZ cells and $8 \mu \mathrm{mol} / \mathrm{L}$ for GBC cells had a more significant apoptosis-inducing effect when compared to the control group. To determine the effect of EVO treatment on genomic 
DNA damage and repair, $\gamma$-H2AX expression was measured by immunofluorescence in GBC-SD cells. EVO increased $\gamma$-H2AX foci in a dose-dependent manner, which suggests EVO promoted cellular genomic DNA damage (Figure 2B).

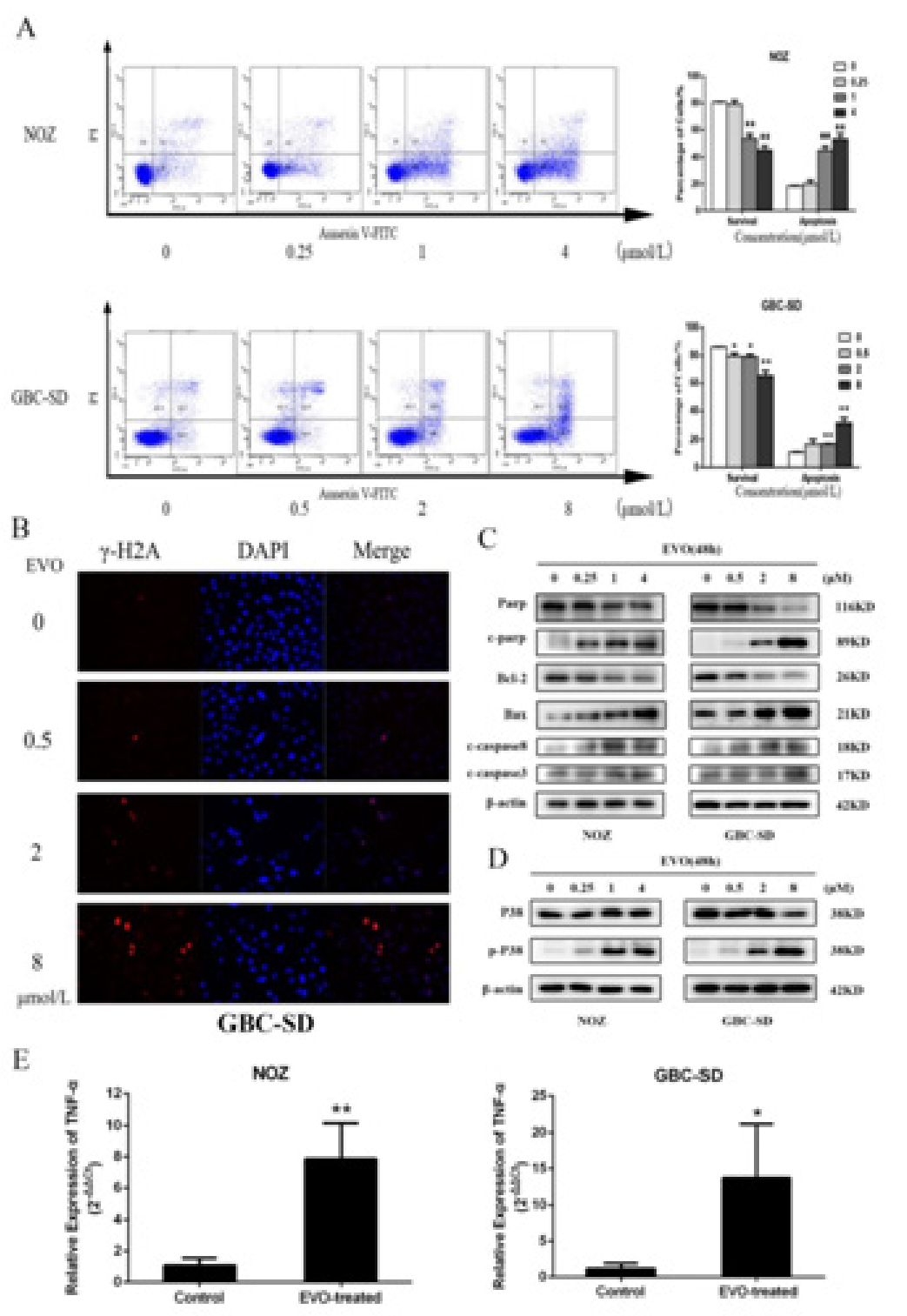

Figure 2: EVO induced NOZ and GBC-SD cells apoptosis through p38 MAPK pathway.

\section{EVO enhanced extrinsic apoptosis by promoting the p38 MAPK-TNF pathway}

To investigate the underlying mechanism of EVO-induced apoptosis, western blot was performed to evaluate the expression of apoptosis-related proteins. Treatment with EVO up-regulated the expression of cleaved caspase-3, cleaved caspase-8, cleaved PARP and Bax while down-regulated the expression of Bcl-2 in a dose-dependent manner (Figure 2C). The decreasing ratio of Bcl2 to Bax advanced cell apoptosis. Caspase- 8 and caspase- 3 bear the central part in extrinsic apoptosis. Activated caspase-8 and caspase- 3 indicated that EVO could promote extrinsic apoptosis in NOZ and GBC-SD cells.
P38 MAPK pathway plays an important role in the growth of various tumors, which is associated with apoptosis, inflammation and invasion. In order to demonstrated whether the p38 MAPK pathway is associated with EVO-induced apoptosis, we estimated the related protein expression by western blot analysis, as shown in Figure 2D, the expression level of p-p38 increased significantly in a dose-dependent manner while the p38 expression had no apparent change in NOZ and minorly decreased in GBC-SD cells which indicated the activation of p38 MAPK pathway to induce extrinsic apoptosis of the gallbladder cancer. Furthermore, tumor necrosis factor- $\alpha(\mathrm{TNF}-\alpha)$, a major mediator of extrinsic apoptosis, may participated in cell apoptosis induced by P38 MAPK pathway. Thus, we evaluated the expression of TNF- $\alpha$ by RT-PCR and found that 
the expression of TNF- $\alpha$ mRNA increased tremendously (Figure $2 \mathrm{E}$ ), which suggests the activation of $\mathrm{p} 38$ MAPK-TNF- $\alpha$ pathway to induce extrinsic apoptosis of the gallbladder cancer cells.

\section{EVO inhibits autophagy by depletion of p53 in GBC-SD and NOZ cells}

Moreover, western blot analysis of autophagy markers was performed to determine the cellular autophagy inhibited by EVO. As shown in Figure 3, western blot revealed that the expression of Beclin-1 was down-regulated while p62 was up-regulated with the increasing concentration of EVO. And the LC3II was decreasing in dose- dependent manner. Furthermore, the depletion of p53 can inhibit autophagy. We also found the level of active p53 was reduced in a dose- dependent manner, which was associated with the inhibition of autophagy induced by EVO. In this part, we demonstrated that EVO attenuated autophagy with specifically autophagy-related proteins, which was regulated by p53/mTORC1associated anti-autophagic pathway.

\section{EVO(48h)}

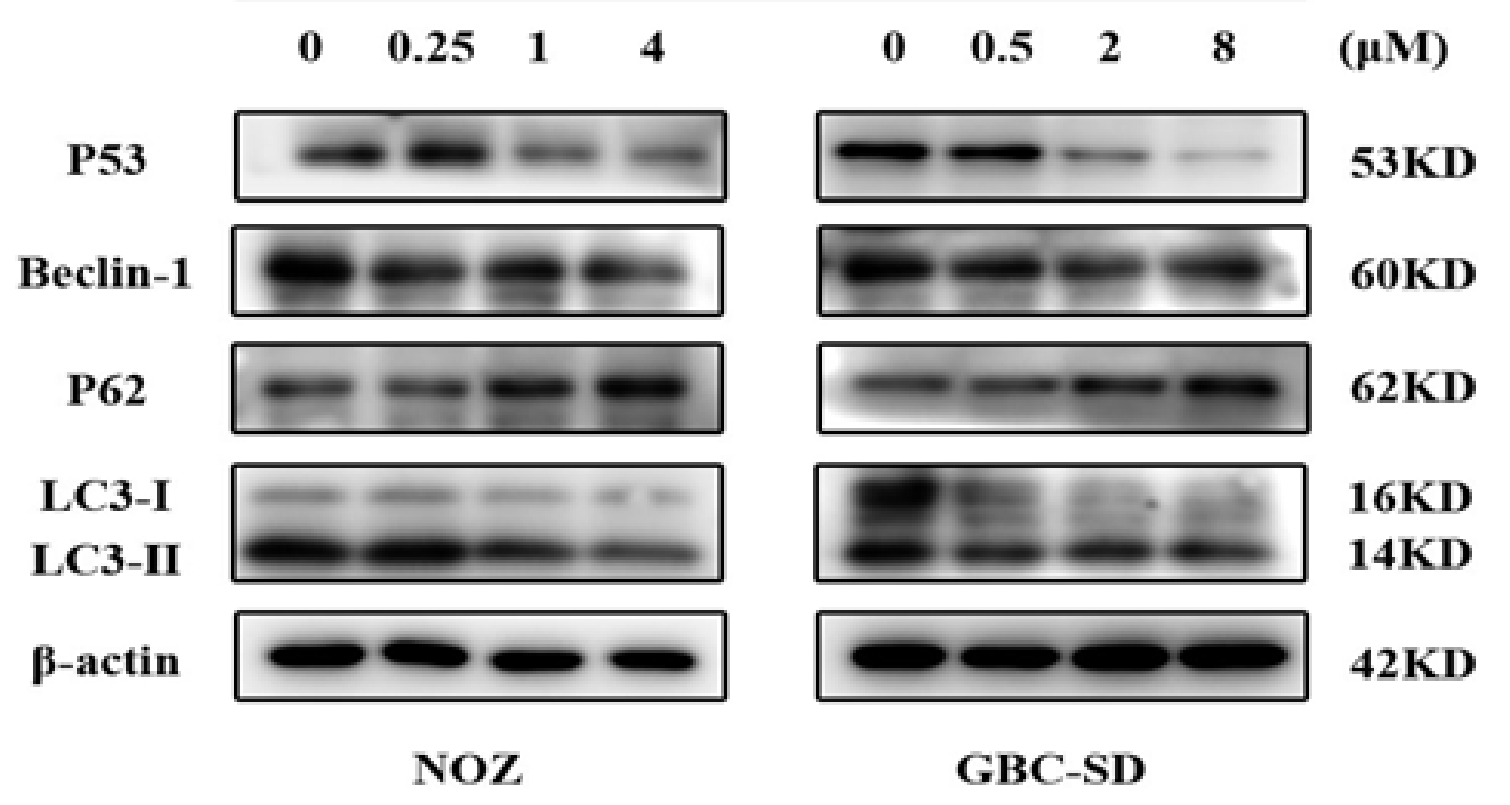

Figure 3: EVO inhibits the autophagy of NOZ and GBC-SD cells.

\section{Discussion}

While the diagnostic and treatment techniques of the gallbladder carcinoma are progressing ceaselessly, there is no effective adjuvant therapy yet. Recent studies have identified that Chinese medicines play a significant part in cancer therapy. Evodiamine has been discovered to have inhibitory effects on certain kinds of cancers. Hence, we investigated the EVO-induced inhibition effects on the carcinoma of the gallbladder and explored the underlying mechanisms. In our study, we found that EVO remarkably inhibited the viability by promoting extrinsic apoptosis with related caspases activation through p38 MAPK pathway and inhibited autophagy in a dose-dependent manner in vitro and vivo. All these experiments above confirmed that the EVO has powerful anti-cancer effects against gallbladder cancer.

P38 MAPK pathway regulates various cellular activities concerned with cancer development, including proliferation, differentiation, apoptosis and inflammation $[27,28]$ Tumor necrosis factor- $\alpha$ (TNF- $\alpha$ ), which is major mediator of extrinsic apoptosis, was initially considered only to be secreted by inflammation-related cells such as macrophages, but more recent research has found that a wide variety of tumor cells can secrete TNF- $\alpha$, including breast, ovarian, colon cancers [29]. P38 MAPK pathway can be activated by various types of cellular stress such as oxidative, genotoxic, and osmotic stress and maybe more importantly by pro-inflammatory cytokines such as TNF- $\alpha$ [27]. p38 MAPK pathway regulates the expression of TNF- $\alpha$. MK2 and MK3 are two downstream kinases phosphorylated and activated by $\mathrm{p} 38$, which phosphorylate mRNAbinding protein tristetraprolin (TTP).

TTP decreases TNF biosynthesis by binding to TNF mRNA and thus suppressing its translation. Phosphorylation of TTP by MK2 neutralizes the function of TTP, which promotes the expression of TNF and triggers the process of extrinsic apoptosis [30], while in the meantime, RT-PCR results exhibit the increased expression of TNF- $\alpha$. Our western blot results show that the expression of p-p38, cleaved-caspase 8 and cleaved-caspase 3 increased in a dose-dependent manner, indicating the activation of p38 MAPKs pathway and extrinsic apoptosis. Therefore, we hypothesize that EVO-induced extrinsic apoptosis may be due to over-expression of TNF initiated by p38 MAPKs. Furthermore, autophagy and apoptosis are controlled by multiple common upstream signals, 
and generally autophagy inhibition can enhance apoptosis [31,32]. Firstly, autophagy usually dismantles the cell before apoptosis, thus inhibition of autophagy can lead to apoptosis when severe stress occurs.

Moreover, autophagy can selectively target pro-apoptotic proteins which have already been ubiquitylated. Such a modification enables them to interact with autophagy receptors, namely adaptors, including p62, which bind with both ubiquitylated substrates and LC3. Thus, inhibition of autophagy can promote apoptosis. For instance, due to the selective removal of cleaved caspase- 8 mediated by autophagy, downregulation of its function enable colon cancer cells and hepatocyte to increase apoptosis by augmentation of caspase- 8 activity $[33,34]$ In our research, the inhibition of autophagy caused by EVO may have the same effect on strengthening apoptosis with up-regulation of cleaved caspase-8 in extrinsic apoptosis pathway. Thirdly, p38 MAPK pathway itself possibly control the balance of apoptosis and autophagy in response to EVO. The p38 MAPK plays a positive role in apoptosis as mentioned above while exerts negative effects in the process of autophagy. It has been proved that suppression of the p38 MAPK pathway promotes autophagic cell death in TNF- $\alpha$-treated L929 cells [35].

Analogously, triterpenes induces autophagy in colon cancer through the inhibition of p38 MAPK[36]. Although the mechanisms between p38 MAPK pathway regulation and autophagy remain largely unknown, the phosphorylation of the autophagy-related 5 (Atg5) at threonine 75 through p38 MAPK may associate with down-regulation of autophagy [37]. In our results, we have found that the inhibition of autophagy is induced by EVO, which was probably due to the phosphorylation of Atg 5 mediated by the activation of p38 MAPK pathway according to the mechanisms mentioned above. On the whole, our study showed that EVO is a potent viability inhibitor of gallbladder cancer cells, and the inhibition was time and dose-dependent. The underlying molecular mechanisms are associated with activation of extrinsic apoptosis regulated by p38 MAPK pathway and inhibition of autophagy through p53-mTORC1 pathway. Furthermore, p38 MAPK may act as a common cross-talk pathway to balance the effect of apoptosis and autophagy. Therefore, we hold a strong belief that EVO could be a novel promising agent for the treatment of gallbladder cancer cells.

\section{Acknowledgment}

This study was supported by the National Natural Science Foundation of China (No. 31620103910, 81272402, 91440203, 81502433 and 3170110831501127 ), the 10th Undergraduate Training Programs for Innovation of Shanghai Jiao Tong University School of Medicine(State Project; 201710248071),the Shanghai Science and Technology Commission Key Basic Research Program (No. 16JC1400200), the Shanghai Key Laboratory of Biliary Tract Disease Research Foundation (17DZ2260200), the Shanghai Sailing Program (16YF1407100), and the Ph.D. Innovation Fund of Shanghai Jiao Tong University School of Medicine to Yuan-yuan Ye (No. BXJ201724).

\section{References}

1. Dwivedi AN, Jain S, Dixit R (2015) Gall bladder carcinoma: Aggressive malignancy with protean loco-regional and distant spread. World J Clin Cases 3(3): 231-244.

2. Shukla SK, Singh G, Shahi KS, Bhuvan, Pant P (2018) Staging, Treatment, and Future Approaches of Gallbladder Carcinoma. J Gastrointest Cancer 49(1): 9-15.

3. Rakic M, Patrlj L, Kopljar M, Klicek R, Kolovrat M, et al. (2014) Gallbladder cancer. Hepatobiliary Surg Nutr 3(5): 221-226.

4. Hundal R, Shaffer EA (2014) Gallbladder cancer: epidemiology and outcome. Clin Epidemio 6: 99-109.

5. Bal MM, Ramadwar M, Deodhar K, Shrikhande S (2015) Pathology of gallbladder carcinoma: current understanding and new perspectives. Pathol Oncol Res 21(3): 509-525.

6. Goetze TO (2015) Gallbladder carcinoma: Prognostic factors and therapeutic options. World J Gastroenterol 21(43): 12211-12217.

7. Tan Q, Zhang J (2016) Evodiamine and Its Role in Chronic Diseases. Adv Exp Med Biol 929: 315-328.

8. Gavaraskar K, Dhulap S, Hirwani RR (2015) Therapeutic and cosmetic applications of Evodiamine and its derivatives-A patent review. Fitoterapia 106: 22-35.

9. Chen TC, Chien CC, Wu MS, Chen YC (2016) Evodiamine from Evodia rutaecarpa induces apoptosis via activation of JNK and PERK in human ovarian cancer cells. Phytomedicine 23(1): 68-78.

10. Hu CY, Wu HT, Su YC, Lin CH, Chang CJ, et al. (2017) Evodiamine Exerts an Anti-Hepatocellular Carcinoma Activity through a WWOX-Dependent Pathway. Molecules 22(7).

11. Sun C, Zhang G, Luan S, Luan C, Shao H, et al. (2016) Evodiamine inhibits the proliferation of leukemia cell line K562 by regulating peroxisome proliferators-activated receptor gamma (PPARgamma) pathway. J Recept Signal Transduct Res 36(4): 422-428.

12. Rasul A, Yu B, Zhong L, Khan M, Yang H, et al. (2012) Cytotoxic effect of evodiamine in SGC-7901 human gastric adenocarcinoma cells via simultaneous induction of apoptosis and autophagy. Oncol Rep 27(5): 1481-1487.

13. Fang C, Zhang J, Qi D, Fan X, Luo J, et al. (2014) Evodiamine induces G2/M arrest and apoptosis via mitochondrial and endoplasmic reticulum pathways in H446 and H1688 human small-cell lung cancer cells. PLoS One 9(12): e115204.

14. Hong JY, Park SH, Min HY, Park HJ, Lee SK (2014) Anti-proliferative effects of evodiamine in human lung cancer cells. J Cancer Prev 19(1): 7-13.

15. Chien CC, Wu MS, Shen SC, Ko CH, Chen CH, et al. (2014) Activation of JNK contributes to evodiamine-induced apoptosis and G2/M arrest in human colorectal carcinoma cells: a structure-activity study of evodiamine. PLoS One 9(6): e99729.

16. Khan M, Bi Y, Qazi JI, Fan L, Gao H (2015) Evodiamine sensitizes U87 glioblastoma cells to TRAIL via the death receptor pathway. Mol Med Rep 11(1): 257-262.

17. Jaiswal PK, Goel A, Mittal RD (2015) Survivin: A molecular biomarker in cancer. Indian J Med Res 141(4): 389-397.

18. Shen H, Zhao S, Xu Z, Zhu L, Han Y, et al. (2015) Evodiamine inhibits proliferation and induces apoptosis in gastric cancer cells. Oncol Lett 10(1): 367-371.

19. Bai X, Meng H, Ma L, Guo A (2015) Inhibitory effects of evodiamine on human osteosarcoma cell proliferation and apoptosis. Oncol Lett 9(2): 801-805. 
20. Zhang T, Qu S, Shi Q He D, Jin X (2014) Evodiamine induces apoptosis and enhances TRAIL-induced apoptosis in human bladder cancer cells through mTOR/S6K1-mediated downregulation of Mcl-1. Int J Mol Sci 15(2): 3154-3171.

21. Tu YJ, Fan X, Yang X, Zhang C, Liang HP (2013) Evodiamine activates autophagy as a cytoprotective response in murine Lewis lung carcinoma cells. Oncol Rep 29(2): 481-490.

22. Liu AJ, Wang SH, Chen KC, Kuei HP, Shih YL, et al. (2013) Evodiamine, a plant alkaloid, induces calcium/JNK-mediated autophagy and calcium/ mitochondria-mediated apoptosis in human glioblastoma cells. Chem Biol Interact 205(1): 20-28.

23. Zhao LC, Li J, Liao K, Luo N, Shi QQ et al. (2015) Evodiamine Induces Apoptosis and Inhibits Migration of HCT-116 Human Colorectal Cancer Cells. Int J Mol Sci 16(11): 27411-27421.

24. Liu M, Wilson NO, Hibbert JM, Stiles JK (2013) STAT3 regulates MMP3 in heme-induced endothelial cell apoptosis. PLoS One 8(8): e71366.

25. Wei L, Jin X, Cao Z, Li W (2016) Evodiamine induces extrinsic and intrinsic apoptosis of ovarian cancer cells via the mitogen-activated protein kinase/phosphatidylinositol-3-kinase/protein kinase B signaling pathways. J Tradit Chin Med 36(3): 353-359.

26. Wei WT, Chen H, Wang ZH, Ni ZL, Liu HB, et al. (2012) Enhanced antitumor efficacy of gemcitabine by evodiamine on pancreatic cancer via regulating PI3K/Akt pathway. Int J Biol Sci 8(1): 1-14.

27. Kim EK, Choi EJ (2015) Compromised MAPK signaling in human diseases: an update. Arch Toxicol 89(6): 867-882.

28. Peluso I, Yarla NS, Ambra R, Pastore G, Perry G (2017) MAPK signalling pathway in cancers: Olive products as cancer preventive and therapeutic agents. Semin Cancer Biol S1044-579X (17): 30165-30167.

\section{ISSN: 2574-1241}

DOI: 10.26717/BJSTR.2018.06.001347

Ying-bin Liu. Biomed J Sci \& Tech Res

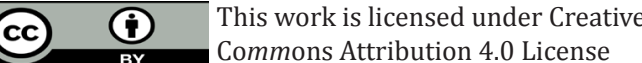

Submission Link: https://biomedres.us/submit-manuscript.php
29. Li J, Zhang Y, Chen L, Lu X, Li Z, et al. (2018) Cervical Cancer HeLa Cell Autocrine Apoptosis Induced by Coimmobilized IFN-gamma plus TNFalpha Biomaterials. ACS Appl Mater Interfaces 10(10): 8451-8464.

30. Menon MB, Gaestel M (2018) MK2-TNF-Signaling Comes Full Circle: Trends Biochem Sci 43(3): 170-179.

31. Thorburn A (2014) Autophagy and its effects: making sense of doubleedged swords. PLoS Biol 12(10): e1001967.

32. Marino G, Niso Santano M, Baehrecke EH, Kroemer G (2014) Selfconsumption: the interplay of autophagy and apoptosis. Nat Rev Mol Cell Biol 15(2): 81-94.

33. Hou W, Han J, Lu C, Goldstein LA, Rabinowich H (2010) Autophagic degradation of active caspase-8: a crosstalk mechanism between autophagy and apoptosis. Autophagy 6(7): 891-900

34. Amir M, Zhao E, Fontana L, Rosenberg H, Tanaka K, et al. (2013) Inhibition of hepatocyte autophagy increases tumor necrosis factordependent liver injury by promoting caspase-8 activation. Cell Death Differ. 20(7): 878-887.

35. Ye YC, Yu L, Wang HJ, Tashiro S, Onodera S, et al. (2011) TNFalphainduced necroptosis and autophagy via supression of the p38-NFkappaB survival pathway in L929 cells. J Pharmacol Sci 117(3): 160-169.

36. Thyagarajan A, Jedinak A, Nguyen H, Terry C, Baldridge LA, et al. (2010) Triterpenes from Ganoderma Lucidum induce autophagy in colon cancer through the inhibition of p38 mitogen-activated kinase (p38 MAPK). Nutr Cancer 62(5): 630-640.

37. Keil E, Hocker R, Schuster M, Essmann F, Ueffing N, et al. (2013) Phosphorylation of Atg5 by the Gadd45beta-MEKK4-p38 pathway inhibits autophagy. Cell Death Differ 20(2): 321-332.

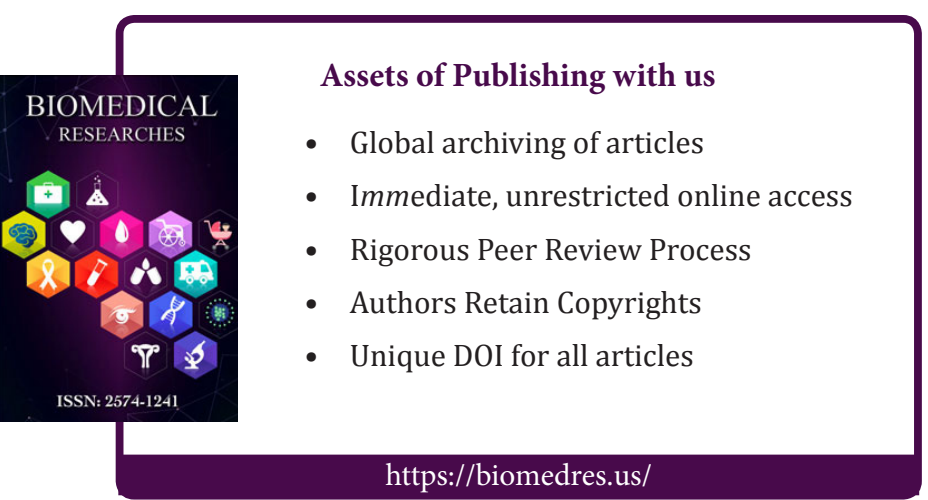

Cite this article: Jun j X, R Y, Fang j Y,Ying b L. Evodiamine Induces Extrinsic Apoptosis Through P38 Mapk Pathway and Inhibits Autophagy 